\title{
Editorial: Microbial Drivers of Sociality - From Multicellularity to Animal Societies
}

\author{
Peter H. W. Biedermann ${ }^{1 *}$, Marko Rohlfs ${ }^{2 *}$, Dino P. McMahon ${ }^{3,4 *}$ and Joël Meunier ${ }^{5 *}$ \\ ${ }^{1}$ Chair of Forest Entomology and Protection, University of Freiburg, Freiburg, Germany, ${ }^{2}$ Population and Evolutionary \\ Ecology Group, Institute of Ecology, University of Bremen, Bremen, Germany, ${ }^{3}$ Institut für Biologie, Freie Universität Berlin, \\ Berlin, Germany, ${ }^{4}$ Department for Materials and the Environment, Bundesanstalt für Materialforschung und -prüfung (BAM), \\ Berlin, Germany, ${ }^{5}$ Institut de Recherche sur la Biologie de I'Insecte, UMR 7261 CNRS - Université de Tours, Tours, France
}

Keywords: microbe, sociality, multicellularity, evolution, symbiosis

\section{Editorial on the Research Topic}

Microbial Drivers of Sociality - From Multicellularity to Animal Societies

\section{MICROBES-AN ECOLOGICAL DRIVER OF SOCIAL EVOLUTION}

While sociality is present in a taxonomically diverse number of species, most animals remain solitary (Bourke, 2011). Over the last centuries, this apparent imbalance in social and non-social animals has led to a great deal of research aimed at shedding light on the biotic and abiotic factors explaining the emergence and maintenance of sociality in nature (West et al., 2015). Among them, microbes were quickly identified as a major problem for the evolution of social life, because frequent contact between group members typically facilitates the transmission of pathogens, high nest fidelity favours the establishment of microbial pathogens close to their social hosts and, finally, because social groups often exhibit limited genetic diversity and thus limited genetic resistance against certain pathogen strains (Schmid-Hempel, 1998; Cremer et al., 2007). However, this long-standing view has changed considerably over the last few years. Recent research indeed revealed that group living may be more effective than solitary living to limit the risk of infection by pathogenic microbes because group living also allows the development of an additional layer of defence against pathogens in the form of social immunity (Cremer et al., 2007; Cotter and Kilner, 2010). Under strong pressure from pathogens, microbes could therefore promote, rather than hinder, the evolutionary transition from solitary to group living (Meunier, 2015; Biedermann and Rohlfs, 2017). Moreover, we are increasingly aware that many microbes provide essential benefits to their hosts by performing critical digestive, physiological, and reproductive functions (Engel and Moran, 2013; McFall-Ngai et al., 2013). The need to access beneficial microbes may thus have played a role in the expression of frequent and tight interactions between conspecifics and ultimately promoted social evolution (Wilson, 1971; Onchuru et al., 2018). Finally, a growing number of studies suggest that microbes could enforce the aggregation and expression of cooperative behaviours of the hosts to increase their chance of reaching new hosts and may therefore be involved in the evolution of host sociality (Lewin-Epstein et al., 2017) (but see Johnson and Foster, 2018).

In this Research Topic, we aimed to provide an overview of these recent advances and the potential limitations of our understanding of the roles of microbes in the social evolution of hosts. The collection of articles presented here responds to these objectives by focusing on five major points: (1) a potential limit in our understanding of the roles of microbes in social evolution comes from the multiple definitions of sociality and the persistent boundaries between research communities, (2) the access to social immunity does not necessarily modify investment into 
individual immunity, (3) further researches are needed to explore the wide diversity of social immunity in non-eusocial species and to shed light on the mechanisms of recognition of microbial pathogens in social groups, (4) habitat quality can be a prime candidate to explain the association between microbes and social evolution, and (5) the necessity to acquire symbionts from conspecifics could be a key evolutionary driver of sociality. In the following parts, we will briefly summarise how the studies contained in this Research Topic address these five points and then conclude on potential future directions of research.

\section{On the Multiple Definitions of Sociality and the Necessity to Break Boundaries Between Research Communities}

Social life is not only present in various organisms but also in all kinds of forms: simple aggregations of individuals, facultative or obligatory parental care, cooperatively breeding groups and super-organismal eusocial societies (Bourke, 2011). However, research on animal sociality has long focused exclusively on the fascinating eusocial organisation of certain hymenopteran and isopteran insects, hampering our general understanding of the evolution of all forms of social life in nature (Elgar, 2015; Meunier and Steiger, 2018). Somewhat surprisingly, the results of our call for this Research Topic suggest that this bias is being corrected: 7 of the 16 published articles are based on noneusocial systems (Table 1). All else being equal, this indicates that the research community studying the link between microbes and social evolution has moved away from exclusive eusocial model systems. However, the results of our call also point out existing partitions between communities, as we only managed to receive two non-insect articles and unfortunately none on a noneukaryotic system (Table 1). This bias may come from the fact that the editors of this special issue are mostly working on insect models, and have failed to reach out and/or to attract members of the other communities. This bias may also stem from the multiple definitions of sociality present in the literature (Rubenstein and Abbot, 2017), which are often very specific to each community, and which may have-for instance-excluded vast communities of researchers studying family life and aggregation behaviours much more frequent in non-insects species.

\section{On the Links Between Individual and Social Immunities}

A long-standing question about the link between microbial pathogens and social evolution is whether access to additional defences against microbial pathogens (i.e., social immunity) necessarily comes with reduced investment in individual immunity. Five manuscripts of this Research topic illustrate the complexity of this question and the diversity of answers that can be given. First, Meusemann et al. used genomic and transcriptomic data from eight termite species, representing wood-dwelling and foraging species, plus 14 other winged insects (Pterygota) and found that differences in the types of sociality do not reflect differences in the intensity of natural selection on immune genes. Instead, they found evidence for a genomewide pattern of relaxed selection on these genes in termites.
Second, Baeuerle et al. used experimental approaches on 14 bumblebee species to show that the investment in an external defence against pathogens (in the venom) does not trade-off against the investment in internal immune defence present in the hemolymph. Third, Esparza-Mora et al. revealed in a termite that inhibiting an external enzyme capable of degrading entomopathogenic fungi does not trigger collective defences such as allogrooming, but instead reduce defensive cannibalistic behaviours. This suggests that the individual immune system is linked to certain collective immune behaviours in this termite. Fourth, Cole and Rosengaus emphasised the importance of social environment on pathogen resistance, as they showed that the presence of a king may help to mitigate the negative effects of a queen's infection during colony foundation in a dampwood termite. Finally, Pull and McMahon propose a comprehensive review of social immunity and emphasise that "superorganism immunity" may fulfil an analogous function to the immune system of Metazoa.

\section{On the Diversity of Social Immunity and Mechanisms of Recognition of Microbial Pathogens}

The recent proposal that social immunity is not specific to eusocial systems (Cotter and Kilner, 2010; Van Meyel et al., 2018) opened numerous questions about the diversity of its forms, the type of immune benefits provided by the social environment and the mechanisms mediating the recognition of pathogenic microbes in social species. Two manuscripts of this Research Topic offer an overview of these questions. First, Trienens and Rohlfs investigated forms of social immunity in groups of fruit fly larvae. They showed that larvae suppress the invasion of a harmful fungus by the summative effect of individuals at high densities and that larger groups of larvae at the same density can control fungal growth more efficiently. This indicates a potential collective defence against habitat invasion by pathogenic fungi in insects that exhibit mere aggregation behaviour. Second, Goes et al. reviewed the literature on leaf-cutting ants' social immunity to investigate how workers protect their fungal garden against harmful microbes. They reveal that workers discriminate against harmful microbes via chemical cues originating from the antagonistic microbe and/or semiochemicals released by the fungus-garden during harmful interactions, as well as via associative learning when workers connect the microbe cues with damage in the fungus garden.

\section{On the Key Role of Habitat Quality in the Association Between Microbes and Social Evolution}

Several articles dealt with the issue that habitat can shape interactions of animals with microbes, which in turn affects the animal's social behaviour. Three of these articles specifically looked at wood as a substrate for insects, which is acknowledged to be very favourable for the evolution of sociality because of its structural resistance and longevity compared to the life of an insect (Hamilton, 1978; Kirkendall et al., 2015). In a review article, Dillard and Benbow argue that two additional factors may 
TABLE 1 | Articles included in our Research Topic.

\begin{tabular}{|c|c|c|c|}
\hline References & Type & Study organism(s) & Level of sociality \\
\hline Cole and Rosengaus & Research article & Zootermopsis angusticollis (Blattodea) & Eusociality \\
\hline Meusemann et al. & Research article & Various Blattodea & Various levels of sociality \\
\hline Tragust et al. & Research article & Lasius niger (Hymenoptera) & Eusociality \\
\hline Baeuerle et al. & Research article & 14 species of bumblebees (Hymenoptera) & Eusociality \\
\hline Sinotte et al. & Review & Eusocial insects in general & Eusociality \\
\hline Esparza-Mora et al. & Research article & Reticulitermes flavipes (Blattodea) & Eusociality \\
\hline Figueiredo and Kramer & Review & Animals in general & All levels of sociality \\
\hline Nalepa & Review & Lower termites and Cryptocercus spp. (Blattodea) & Eusociality and subsociality \\
\hline Dunn et al. & Review & Hominins & Different levels of sociality \\
\hline Bratburd et al. & Review & Social insects & Different levels of sociality \\
\hline Trienens and Rohlfs & Research article & Drosophila melanogaster (Diptera) & Aggregation \\
\hline Goes et al. & Review & Attini (Hymenoptera) & Eusociality \\
\hline Biedermann & Research article & Xyleborus affinis (Coleoptera) & Cooperatively breeding \\
\hline Pull and McMahon & Review & Eusocial insects in general & Eusociality \\
\hline Dillard and Benbow & Review & Various wood-dwelling insects & Different levels of sociality \\
\hline Nuotclà et al. & Research article & Xyleborinus saxesenii (Coleoptera) & Cooperatively breeding \\
\hline
\end{tabular}

facilitate prolonged parent-offspring contact and cooperative behaviours in insects. First, the low nutritional value of wood selects for associations with nutrient-enriching microbes that need to be transferred to offspring, which is often through social contact. Furthermore, insects compete with many antagonistic microbes in this habitat and collective defence is often better to keep them in cheque. A research study by Biedermann found evidence for collective pathogen defence in a cooperatively breeding, fungus-farming ambrosia beetle. Delayed dispersing female offspring showed more social hygienic behaviour at places within the nest with higher abundances of antagonistic fungi. Nuotclà et al. showed in a closely related ambrosia beetle species with a similar social system that the two nutritionally important fungal mutualists of this species vary in their relative abundance depending on the dryness of the wood substrate. Interestingly this fed back on the social behaviour and the delayed dispersal periods of daughters in this facultatively eusocial beetle. Finally, a study by Tragust et al. on founding Lasius niger ant queens showed that queens exposed to pathogens invest simultaneously in formic acid defence and higher worker production. Surprisingly there was no measurable trade-off between this individual immune defence and reproduction at an early nest stage, but this may have effects on later fitness.

\section{On the Role of Symbionts as Promoters of Hosts' Social Evolution}

The growing awareness that microbes residing on and in a host can provide it with major benefits has recently stimulated a great number of experimental and theoretical research on the impacts of symbionts in the social evolution of hosts. This is illustrated by five articles in this Research Topic. First, Bratburd et al. examined the general role of defensive microbial symbionts in host protection against pathogens in terms of behavioural and immune responses and discussed why insects are good models to study issues relating to human health and agriculture. Second, Dunn et al. used a comparative approach to study how microbiomes of hominins have changed over evolutionary time, questioning their impact on the evolution of several host functions and discussing the possibility that prosocial microbes promoted hominin social behaviour. Third, Nalepa discussed why symbiont transmission via proctodeal trophallaxis and sociality are likely to have entangled evolutionary histories and conclude that the vertical transmission of gut microbes (flagellates) and the origin of host subsociality are two sides of the same coin in termites. Fourth, Sinotte et al. explored the link between the division of labour and symbiosis in social insects. Their review suggests that structured microbiomes have evolved in parallel to social complexity, and predicts that mature social insect colonies with the most extreme division of labour shows the strongest distinction between caste microbiomes. This suggests that caste-specific microbiomes may enhance symbiotic benefits and the efficiency of division of labour. Finally, Figueiredo and Kramer took another perspective and focused on the microbes themselves. Their review describes cooperation and conflict within the microbiota. They discuss how these parameters can affect animal hosts and conclude that an explicit consideration of social dynamics within symbiont communities is crucial to advance our understanding of how microbes shape animal function and evolution.

\section{CONCLUSION}

Overall, this Research Topic emphasises the multiple roles of microbes in the social evolution of their hosts, which range from obstruction to promotion. It also illustrates why groupliving animals specifically face an intense tug-of-war between the necessity to limit the inherently high risk of pathogen infection and transmission within the nest as well as the necessity to protect and efficiently transmit essential symbionts within the nest and to dispersing sexuals. The outcome of this war can 
have profound impacts on the life-history traits of a given species. Finally, it also points out that although our current understanding of the link between microbes and social evolution is based on a wider range of social systems and is thus becoming more comprehensive, it still needs to bring together the results of all research communities studying different organisms. We believe that this Research Topic is a first step toward achieving this goal.

\section{AUTHOR CONTRIBUTIONS}

All authors wrote the manuscript.

\section{REFERENCES}

Biedermann, P. H. W., and Rohlfs, M. (2017). Evolutionary feedbacks between insect sociality and microbial management. Curr. Opin. Insect Sci. 22, 92-100. doi: 10.1016/j.cois.2017.06.003

Bourke, A. F. G. (2011). Principles of Social Evolution. Oxford: Oxford University Press.

Cotter, S. C., and Kilner, R. M. (2010). Personal immunity versus social immunity. Behav. Ecol. 21, 663-668. doi: 10.1093/beheco/arq070

Cremer, S., Armitage, S. A. O., and Schmid-Hempel, P. (2007). Social immunity. Curr. Biol. 17, R693-702. doi: 10.1016/j.cub.2007.06.008

Elgar, M. A. (2015). Integrating insights across diverse taxa: challenges for understanding social evolution. Front. Ecol. Evol. 3:124. doi: $10.3389 /$ fevo.2015.00124

Engel, P., and Moran, N. A. (2013). The gut microbiota of insects diversity in structure and function. FEMS Microbiol. Rev. 37, 699-735. doi: 10.1111/1574-6976.12025

Hamilton, W. D. (1978). "Evolution and diversity under bark," in Diversity of Insect Faunas, eds L. A. Mound and N. Waloff (Oxford: Blackwell), 154-175.

Johnson, K. V., and Foster, K. R. (2018). Why does the microbiome affect behaviour? Nat. Rev. Microbiol. 16, 647-655. doi: 10.1038/s41579-018-0014-3

Kirkendall, L. R., Biedermann, P. H. W., and Jordal, B. H. (2015). "Evolution and diversity of bark and ambrosia beetles," in Bark Beetles: Biology and Ecology of Native and Invasive Species, eds F. E. Vega and R. W. Hofstetter (London: Academic Press), 85-156.

Lewin-Epstein, O., Aharonov, R., and Hadany, L. (2017). Microbes can help explain the evolution of host altruism. Nat. Communi. 8:14040. doi: 10.1038/ncomms14040

McFall-Ngai, M., Hadfield, M. G., Bosch, T. C. G., Carey, H. V., DomazetLošo, T., Douglas, A. E., et al. (2013). Animals in a bacterial world, a new imperative for the life sciences. Proc. Natl. Acad. Sci. U.S.A. 110, 3229-3236. doi: $10.1073 /$ pnas. 1218525110

Meunier, J. (2015). Social immunity and the evolution of group living in insects. Philos. Trans. R. Soc. B Biol. Sci. 370:20140102. doi: 10.1098/rstb.2014.0102

Meunier, J., and Steiger, S. (2018). Editorial overview: Beyond eusocial insects: studying the other social insects to better understand social evolution. Curr. Opin. Insect Sci. 28, vi-viii. doi: 10.1016/j.cois.2018.07.002

\section{FUNDING}

PB was funded by the German Research Foundation (DFG; Emmy Noether Grant No. BI 1956/1-1). JM was funded by the French National Research Agency (ANR; Project MicroSoc).

\section{ACKNOWLEDGMENTS}

We are grateful to Juan Carlos Cambronero Heinrichs, Janina Diehl, Sifat Munim Tanin, Antoine Melet, and Hanna Cho for summarising the important findings of articles in the special issue.

Onchuru, T. O., Martinez, A., Ingham, C. S., and Kaltenpoth, M. (2018). Transmission of mutualistic bacteria in social and gregarious insects. Curr. Opin. Insect Sci. 28, 50-58. doi: 10.1016/j.cois.2018 05.002

Rubenstein, D. R., and Abbot, P. (eds). (2017). Comparative Social Evolution. Cambridge: Cambridge University Press.

Schmid-Hempel, P. (1998). Parasites in Social Insects. Princeton, NJ: Princeton University Press.

Van Meyel, S., Körner, M., and Meunier, J. (2018). Social immunity: why we should study its nature, evolution and functions across all social systems. Curr. Opin. Insect Sci. 28, 1-7. doi: 10.1016/j.cois.2018. 03.004

West, S. A., Fisher, R. M., Gardner, A., and Kiers, E. T. (2015). Major evolutionary transitions in individuality. Proc. Natl. Acad. Sci. U.S.A. 112, 10112-10119. doi: 10.1073/pnas.14214 02112

Wilson, E. O. (1971). The Insect Societies. Cambridge: Belknap Press of Harvard University Press.

Conflict of Interest: The authors declare that the research was conducted in the absence of any commercial or financial relationships that could be construed as a potential conflict of interest.

Publisher's Note: All claims expressed in this article are solely those of the authors and do not necessarily represent those of their affiliated organizations, or those of the publisher, the editors and the reviewers. Any product that may be evaluated in this article, or claim that may be made by its manufacturer, is not guaranteed or endorsed by the publisher.

Copyright (c) 2021 Biedermann, Rohlfs, McMahon and Meunier. This is an openaccess article distributed under the terms of the Creative Commons Attribution License (CC BY). The use, distribution or reproduction in other forums is permitted, provided the original author(s) and the copyright owner(s) are credited and that the original publication in this journal is cited, in accordance with accepted academic practice. No use, distribution or reproduction is permitted which does not comply with these terms. 